\title{
FILTER MEDIAS FROM GRANULATED FOAM-GLASS, PROPERTIES INVESTIGATED FOR WATER TREATMENT POSSIBILITIES
}

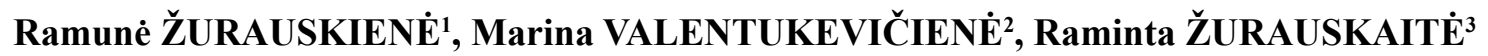 \\ Vilnius Gediminas Technical University, Vilnius, Lithuania \\ E-mails: ${ }^{1}$ ramune.zurauskiene@vgtu.lt; ${ }^{2}$ marina.valentukeviciene@vgtu.lt; ${ }^{3}$ raminta.zurauskaite@stud.vgtu.lt
}

\begin{abstract}
Inorganic material - granulated foam-glass produced from glass breakage in small porous granule shape. Foam-glass is gotten by connecting thoroughly grounded glass with foamers, later this composition is heated in the furnace in especially high temperature and is turned into various diameter greyish granules. Granulated foam-glass is a unique ecological material of which inner structure pores are arranged in such a way that air is trapped inside. Foam-glass production technology is one of the most advanced since the product is made from secondary raw materials, not leaving any third row waste. From granule surface images it can be seen that granule surface has pores and voids, some of these pores are closed, others are connected with granule's inner pores, all pore walls are smooth, and the wall material is vitrified. Pores and voids are arranged chaotically in smaller granules, bigger granules inner space structure is tidy, bigger part is occupied by correctly arranged bigger pores and space between them is filled with smaller pores. Granules are composed of amorphous phase, in mineralogical composition can emit one material cristobalite. In the work were researched two fraction granules: $0 / 2$ and $2 / 4$ as well as determined 0/4 fraction granular-metric composition. Researched and determined main physical granule properties and properties related to water effect to the material, shown in micro-structural granule surface and inner structure images. According to explored properties it can be noted that granules can be adapted in water cleaning technologies.
\end{abstract}

Keywords: absorption, effective granule porosity, foam-glass granules, granule surface structure, porous space reserve.

\section{Introduction}

In the year 2009 production of foam-glass granules started in Lithuania from collected glass waste in the country. Granulated foam-glass - it is an inorganic thermo-insulated material, produced from glass breakage, in small pore granule form. It is unique in that to produce it regular household glass is used. Foam-glass is gotten when connecting grounded to small pieces glass with foamers, later this compound is heated in a furnace in especially high temperature as well as made into various diameter greyish granules. Granulated foam-glass is a unique ecological product produced from waste. Granulated foam-glass is a unique and ecological material which has inner structure pores arranged in such a way that it seems air is held inside. Foam-glass production technology is unique and one of the most advanced since the product is made from secondary raw-recycled materials, not leaving third row waste, in production technology little amount of energy is used due to the equipment adjusted to minimal energy amount usage, and made products fit thermo-insulated and sound isolating needs for buildings, as well as can be used for decorative mortars and other uses. Wide granule size spectrum allows using them in various areas. Granulated foam-glass can be used in such areas like farming, supply systems, oil extraction, bio filtration and other.

Granulated foam-glass is produced into two different types: produced into white and grey foam-glass granules. The grey foam-glass granules are of such fractions: $0 / 2$, $2 / 4,0 / 4,4 / 8,8 / 16 \mathrm{~mm}$, white granules in the factory are divided into more fractions: $0.1 / 0.3,0.25 / 0.5,0.5 / 1,1 / 2$, 2/4, 4/8, 8/16 mm. However when bleaching foam-glass granules are put into chemical compounds that are unfit in water preparation technologies. Different reused filter media materials are in use nowadays (Albalawneh et al. 2017; Sabiri et al. 2017).

Porous granulated foam-glass granule research was carried out in the work. The research used granulated foam-glass granules produced by UAB "Stikloporas" (Druskininkai, Lithuania) production line. The main characteristics of foam-glass granule during the research were explored. It was determined whether grey foam-glass granules fit according to their characteristics related to water effect to material using contaminated water cleaning technologies. 


\section{Methodology}

Separate fraction surface image fixation of porous granulated foam-glass was carried when using different magnification. 2/4, 0/2 $\mathrm{mm}$ fraction granule general view and granule surface image when magnification is 14 and 28 times. $0 / 2$ and $2 / 4$ fraction granule image analyzation was carried out. Granule surface and cut images were gotten with raster electronical microscope. Micro-structure was researched with outside emission scanning electronical microscope SEM JSM-7600F (JEOL). Researched sample cleaving surface when there was lower $(4 \mathrm{kV})$ accelerating voltage. This way an opportunity was formed to research uncovered with electrically conductive layer cleaving surface and also to carry out research on changes that appear in chosen surface place due to heating.

Analysis of granule material mineralogical was carried out by $x$-ray research method using diffractometer DRON7. Anode $-\mathrm{Cu}$, filter $-\mathrm{Ni}$ were used, anode voltage $-30 \mathrm{kV}$, anode current $12 \mathrm{~mA}$, goniometer gaps $(0.5 ; 1.0 ; 1.5) \mathrm{mm}$. ICDD database for x-ray decryption was used. Different fraction granules were explored.

From the factory there were also gotten a granule mix which particle size was $0-4 \mathrm{~mm}$, granule granular-metric composition was determined by sieving through 0.063 , $0.125,0.25,0.5,1,2,4 \mathrm{~mm}$ sieves.

When determining physical properties of granule foam-glass granules were dried and easily poured different fraction granule bulk volume was determined according to the LST EN 1097-3 "Filler mechanical and physical property determining methods. Part 3. Bulk volume and void determination". Granules were submerged in water until they reached constant mass. By submerging soaked granules into water occupied granule volume and granule density were determined. According to these indicators (LST EN 1097-3:2002) separate fraction granule void was calculated. Selected granules were grinded in laboratory mortar and pestle to such small size that sieved material could pass through sieve 008 and composing materials density was determined by drying shrinkage method.

Separate granule fractions were poured into porous meshes from polyamide, weighted and submerged in $(20 \pm 2)^{\circ} \mathrm{C}$ temperature water, after $1,3,5,10 \mathrm{~min}$ and 24 , $48,72,96 \mathrm{~h}$ meshes were taken out from water and, after waiting for excess water to run off, were weighted. Granule separate fraction absorption was calculated by percent according to mass. Granule absorption according to mass was recalculated to granule absorption according to volume.

When determining foam-glass granule absorption by vacuuming, separate granule fractions were poured into porous meshes from polyamide, weighed and put into vacuum utensil (desiccator). System was vacuumed from $0.98 \mathrm{~atm}$. After 60 min vacuuming desiccator was filled with water of 25-30 ${ }^{\circ} \mathrm{C}$ temperature until the samples were completely submerged. After 60 min air was released into the system. Submerged water granules were left for $24 \mathrm{~h}$. After $24 \mathrm{~h}$ granules were taken out of water and, after letting excess water to run off, were weighted.

\section{Results}

Foam-glass granules, which are of 0/2, 2/4 mm fractions, surface and inner structure images are shown in Figures 1-3. By analysing granule outer and inner images such conclusion can be made: granule exterior is covered in small particles which are of plate shape, hexagon form. These particles have width from 0.5 to $3 \mu \mathrm{m}$, while thickness is up to $0.1 \mu \mathrm{m}$. Granule interior is a big amount of bigger and smaller pores. These pores are usually of perfect shape; pore walls are smooth, vitrified. Bigger granule $2 / 4 \mathrm{~mm}$ fraction inner pore structure is tidier, less chaotic and has less smaller granules $-0 / 2 \mathrm{~mm}$. Bigger granule frame is composed of big round pores while voids between them is filled with smaller pores (Zurauskiene 2013).
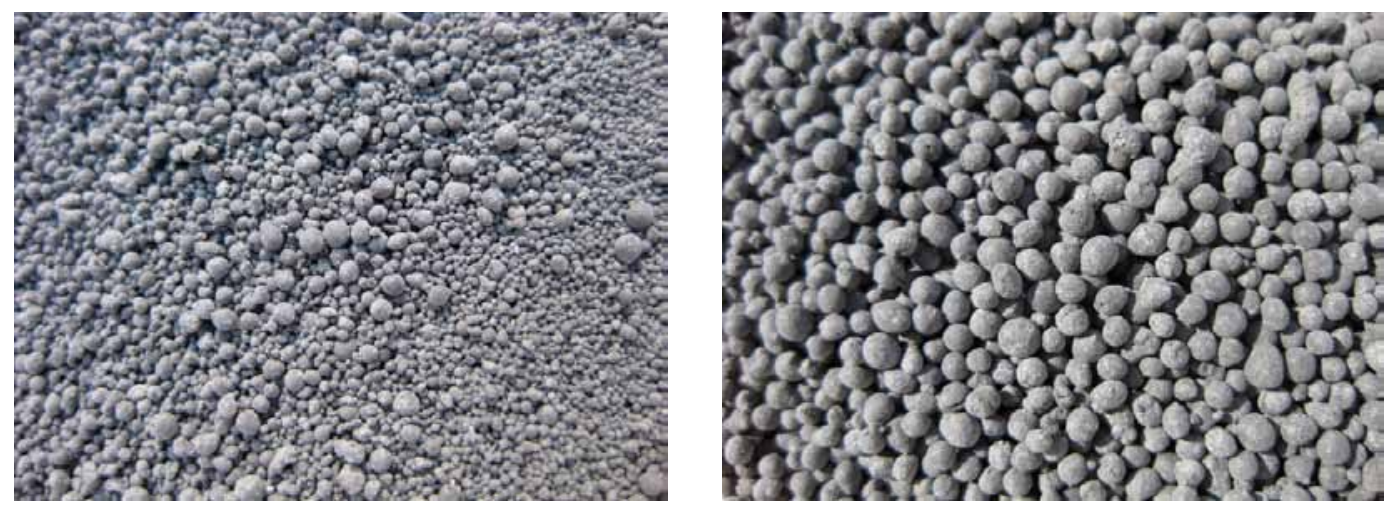

Fig. 1. General granule image: 0/2 fraction (left), 2/4 fraction (right)

1 pav. Granulių vaizdas: 0/2 frakcijos (kairèje), 2/4 frakcijos (dešinejje) 

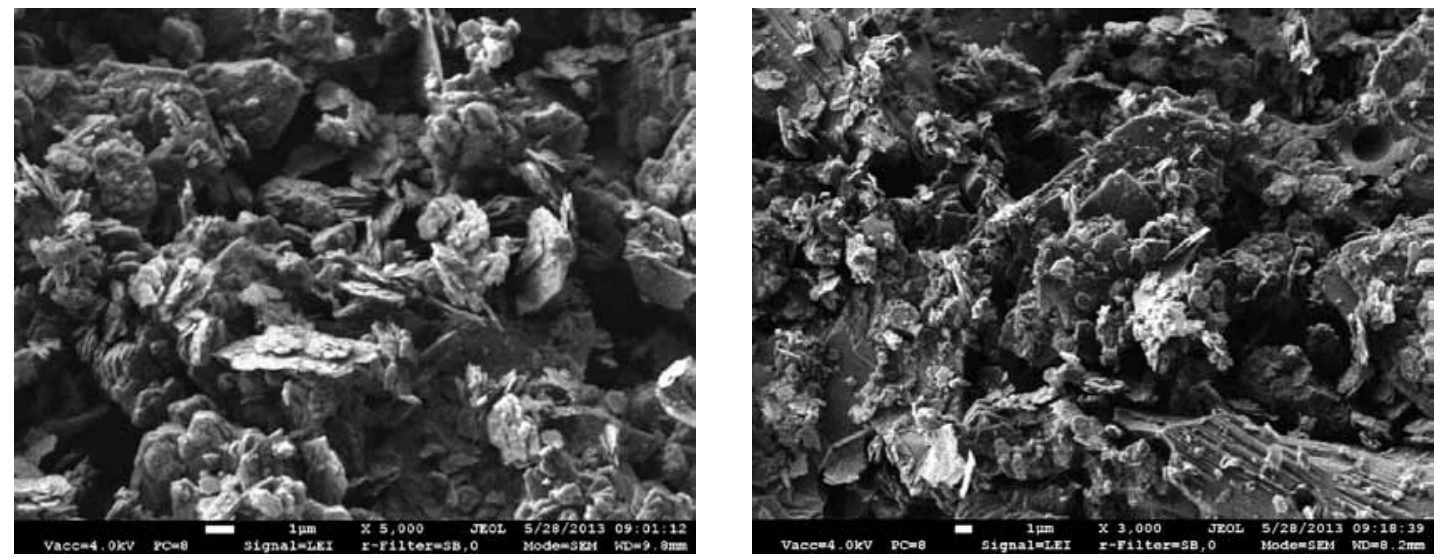

Fig. 2. Foam-glass granules separate fraction surface image: 0/2 fraction, magnification 5000 times (left); 2/4 fraction, magnification 3000 times (right)

2 pav. Atskirų putstiklio granulių frakcijų paviršiaus vaizdas: 0/2 frakcijos, esant 5000 kartų didinimui (kairejje), 2/4 frakcijos, esant 3000 kartų didinimui (dešinèje)
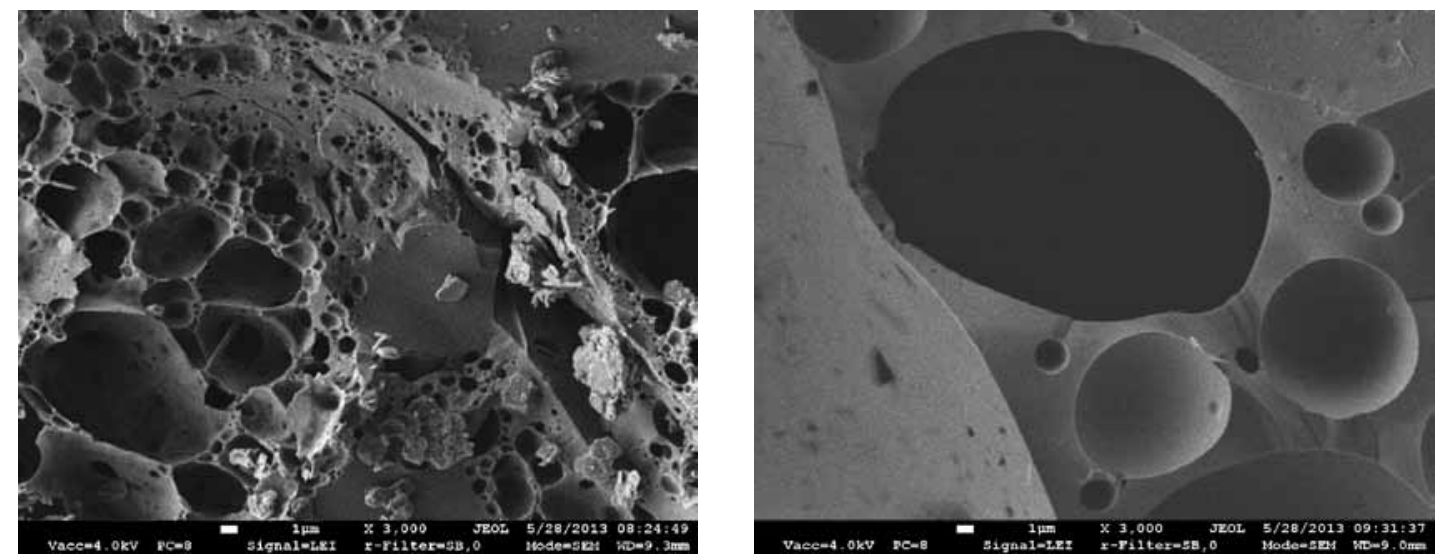

Fig. 3. Foam-glass granules inner structure image, magnification 3000 times: $0 / 2$ fraction (left); $2 / 4$ fraction (right)

3 pav. Atskirų putstiklio granulių frakcijų lūžio vaizdas, esant 3000 kartų didinimui: 0/2 frakcijos (kairèje), 2/4 frakcijos (dešinèje)

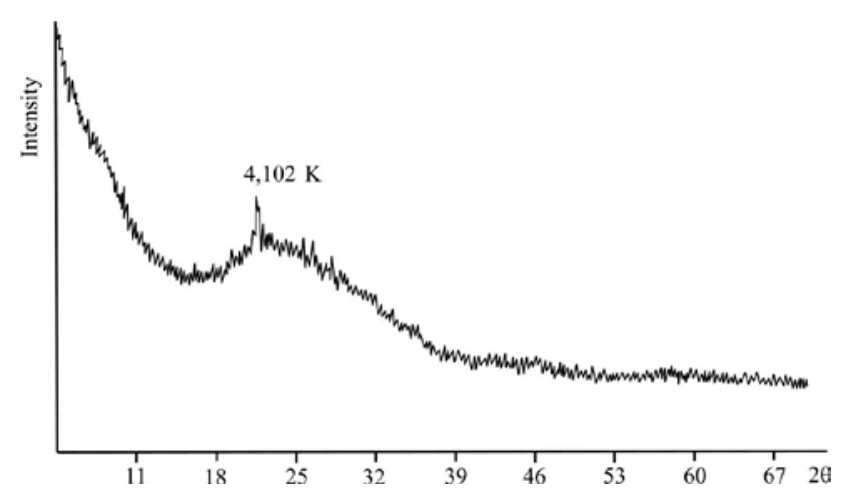

Fig. 4. Granulated foam-glass granule $(2 / 4 \mathrm{~mm})$ material mineralogical composition

4 pav. Putstiklio granulių $(2 / 4 \mathrm{~mm})$ mineraloginè sudètis

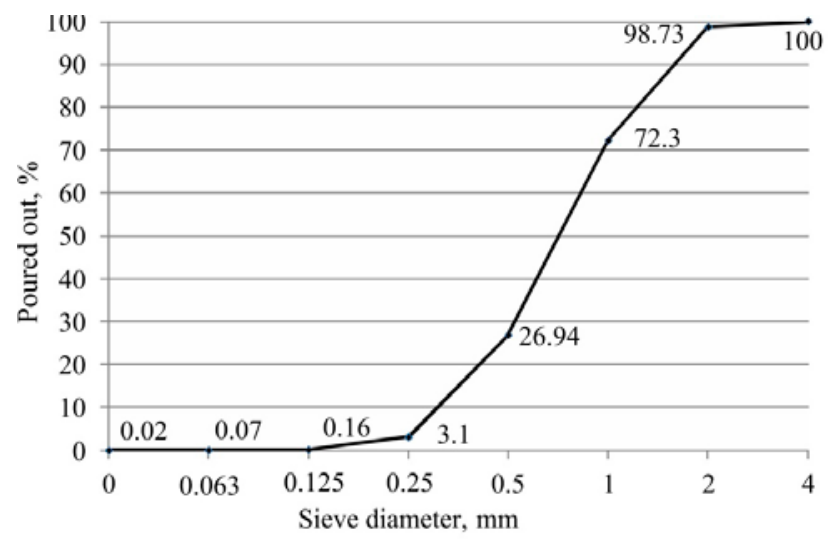

Fig. 5. Foam-glass granule mixture $0-4 \mathrm{~mm}$ granular-metric composition curve

5 pav. Putstiklio granulių 0-4 mm mišinio granuliometrinè sudètis 
X-ray research results are shown in Figure 4. By analysing research results it can be noted that granules consist of amorphous material in which a small cristobalite peak can be emitted. In small granules cristobalite peak has a slightly higher intensity than determined when researching bigger granules.

$0 / 4 \mathrm{~mm}$ fraction mixture produced in the factory was researched determining this mixture granular-metric composition (Figure 5). From 0/4 mm fraction granule granular-metric composition curve (Fig. 4) we can see that main part of granules $45.4 \%$ is composed of granules from 0.5 to $1 \mathrm{~mm}$ size, smaller granules like $0.25 \mathrm{~mm}$ compose only $2.9 \%$, and granules bigger than $2 \mathrm{~mm}$ only compose $1.3 \%$.

Foam-glass granule bulk density, granule density, voids and material density is shown in Table 1.

Foam-glass granule absorption results, when there are different submerging durations, are shown in Table 2, as well as Table 3 shows and recalculated granule absorption results according to volume.
According to absorption research results it can noted that granule absorption depends on foam-glass granule fractions. According to granule volume less water gets absorbed by bigger granules, while biggest absorption is of granules that are composed of smallest fraction grains and have in their composition lots of small particles, that's $0 / 2 \mathrm{~mm}$ fraction particles. According to absorption (absorption according to volume) research results it can be concluded that the bigger particle specific surface area, the more water the particle absorbs - particle surface becomes covered in water and water gets into surface pores and stays in particle surface disparities.

Granule absorption by vacuuming them is shown in Table 4. Porous space reserve show that granule inner open space part, which when submerged in water under normal conditions at the start does not fill with water, however, granules when affected by long-term submerging process gradually filled with water (Zurauskiene et al. 2017).

Water infiltrates into foam-glass granules during vacuuming through surface open pores and gets into deeper

Table 1. Foam-glass granule physical properties

1 lentelè. Putstiklio granulių fizikinès savybès

\begin{tabular}{|l|l|l|l|l|l|}
\hline \multirow{2}{*}{$\begin{array}{c}\text { Foam-glass granule } \\
\text { fraction }\end{array}$} & \multicolumn{5}{|c|}{ Physical properties } \\
\cline { 2 - 6 } & $\begin{array}{c}\text { Bulk density, g/ } \\
\mathrm{cm}^{3}\end{array}$ & $\begin{array}{c}\text { Particle density, g/ } \\
\mathrm{cm}^{3}\end{array}$ & Fraction's void, \% & Fraction's porosity, \% & $\begin{array}{c}\text { Material density, g/ } \\
\mathrm{cm}^{3}\end{array}$ \\
\hline $0 / 2$ & 0.493 & 0.857 & 42.5 & 73.4 & 1.85 \\
\hline $2 / 4$ & 0.195 & 0.364 & 46.3 & 89.5 & \\
\hline
\end{tabular}

Table 2. Foam-glass granule absorption according to mass determining results

2 lentelè. Putstiklio granulių absorbcijos pagal masę rezultatai

\begin{tabular}{|l|l|l|l|l|l|l|l|r|}
\hline \multirow{2}{*}{$\begin{array}{c}\text { Foam-glass granule } \\
\text { fraction }\end{array}$} & \multicolumn{9}{|c|}{ Submerging duration } \\
\cline { 2 - 11 } & $1 \mathrm{~min}$ & $3 \mathrm{~min}$ & $5 \mathrm{~min}$ & $10 \mathrm{~min}$ & $24 \mathrm{~h}$ & $48 \mathrm{~h}$ & $72 \mathrm{~h}$ & $96 \mathrm{~h}$ \\
\hline $0 / 2$ & 25.17 & 26.36 & 27.26 & 27.80 & 31.06 & 35.07 & 35.97 & 37.86 \\
\hline $2 / 4$ & 33.39 & 33.77 & 33.95 & 35.11 & 38.73 & 46.80 & 53.11 & 59.24 \\
\hline
\end{tabular}

Table 3. Foam-glass granule absorption according to volume determination results

3 lentelè. Putstiklio granulių absorbcijos pagal tūrị rezultatai

\begin{tabular}{|l|l|l|l|l|l|l|l|l|}
\hline \multirow{2}{*}{$\begin{array}{c}\text { Foam-glass granule } \\
\text { fraction }\end{array}$} & \multicolumn{9}{|c|}{ Submerging duration } \\
\cline { 2 - 11 }$y$ & \multicolumn{1}{|c|}{$1 \mathrm{~min}$} & \multicolumn{1}{c|}{$3 \mathrm{~min}$} & $5 \mathrm{~min}$ & $10 \mathrm{~min}$ & $24 \mathrm{~h}$ & $48 \mathrm{~h}$ & $72 \mathrm{~h}$ & $96 \mathrm{~h}$ \\
\hline $0 / 2$ & 12.41 & 12.99 & 13.44 & 13.70 & 15.31 & 17.29 & 17.73 & 18.67 \\
\hline $2 / 4$ & 6.51 & 6.58 & 6.62 & 6.85 & 7.55 & 9.13 & 10.36 & 11.55 \\
\hline
\end{tabular}

Table 4. Foam-glass granule absorption after vacuuming

4 lentelè. Putstiklio granulių absorbcijos rezultatai po vakuumavimo

\begin{tabular}{|l|l|l|l|l|}
\hline \multirow{2}{*}{\begin{tabular}{c} 
Foam-glass granule $\begin{array}{c}|c| \\
\text { fraction }\end{array}$ \\
\cline { 2 - 6 }
\end{tabular}} & $\begin{array}{c}\text { Granule absorption after } \\
\text { vacuuming them, mass \% }\end{array}$ & $\begin{array}{c}\text { Effective granule porosity, } \\
\%\end{array}$ & $\begin{array}{c}\text { General granule porosity, } \\
\%\end{array}$ & Porous space reserve, \% \\
\hline $0 / 2$ & 38.06 & 30.83 & 32.62 & 5.49 \\
\hline $2 / 4$ & 65.70 & 19.33 & 23.92 & 19.17 \\
\hline
\end{tabular}


connected pores. Porous space reserve shows that how much empty space that is connected through connected pores and capillaries in researched material. This space when affecting granules with water or water solutions slowly fills with water.

\section{Conclusions}

1. From granule surface images we can see that granule surface has pores and voids, some of these pores are closed, others are connected with granule's inner pores, all pore walls are smooth, wall material is vitrified. In smaller granules pores and voids are arranged chaotically, bigger granule inner space structure is tidy, bigger part is occupied by properly arranged bigger pores and space between them is filled with smaller pores.

2. Granules are composed of amorphous phase, can emit one mineral cristobalite of which small amount can be found both in smaller and bigger granules.

3. Researched granule main properties: $0 / 2 \mathrm{~mm}$ fraction: density $-493 \mathrm{~kg} / \mathrm{m}^{3}$, absorption after 96 hour absorption $-37.86 \%$, porous space reserve $-5.49 ; 2 / 4 \mathrm{~mm}$ fraction: density $-195 \mathrm{~kg} / \mathrm{m}^{3}$, absorption after 96 hour absorption $-59.24 \%$, porous space reserve -19.17 ; mixture $0 / 4 \mathrm{~mm}$ fraction: density $-269 \mathrm{~kg} / \mathrm{m}^{3}$.

\section{References}

Albalawneh, A.; Chang, TK.; Alshawabkeh, H. 2017. Greywater treatment by granular filtration system using volcanic tuff and gravel media, Water Science and Technology 75(10): 2331-2341. https://doi.org/10.2166/wst.2017.102

LST EN 1097-3:2002. Filler mechanical and physical property determining methods. Part 3. Bulk volume and void determination. Lithuanian Standards Board, Vilnius: 14.

Sabiri, NE.; Monnier, E.; Raimbault, V.; Masse, A.; Sechet, V.; Jaouen, P. 2017. Effect of filtration rate on coal-sand dual-media filter performances for microalgae removal, Environmental Technology 38(3): 345-352. https://doi.org/10.1080/09593330.2016.1193224

Zurauskiene, R. 2013. Porous granular cellular glass fills the properties and use of mortar. Custom-made to order VP2-
1.3-ÜM-05-K means by "INOCEKIAI LT" according to the contract Nr.9431(4581-MI) [online], [cited 20 November 2015]. Available from Internet: http://www.stikloporas. lt/Naujienos/Projekto-vykdymas-Poringojo-granuliuotoputstiklio-uzpildo-savybiu-nustatymas-ir-panaudojimasskiedinyje-Nr.-VP2-1.3-UM-05-K-01-113

Zurauskiene, R.; Valentukeviciene, M.; Boussouga, Y. A. 2017. Filter medias from recycled concrete, properties investigated for sorption processes, in "Environmental Engineering" 10th International Conference in Vilnius Gediminas Technical University, Lithuania, 27-28 April 2017.

\section{PUTSTIKLIO GRANULIŲ FILTRAVIMO GALIMYBIŲ TYRIMAS PANAUDOJANT JAS VANDENS VALYMO TECHNOLOGIJOSE}

\section{R. Žurauskienė, M. Valentukevičienė, R. Žurauskaitė}

\section{Santrauka}

Neorganinè medžiaga - granuliuotas putstiklis - gaminama iš stiklo duženu, nedideliu porètų granulių pavidalu. Putstiklis gaunamas sujungiant smulkiai sumaltą stiklą su putokšliais, vẻliau šis junginys kaitinamas krosnyse ypač aukštoje temperatūroje bei paverčiamas įvairaus skersmens pilkšvomis granulemis. Granuliuotas putstiklis yra unikali ekologiška medžiaga, kurios vidineje struktūroje poros išdèstytos taip, kad oras yra lyg sulaikomas viduje. Putstiklio gamybos technologija yra viena iš pažangiausių, nes produktas gaminamas iš antrinių žaliavų, nepaliekant trečios eilès atliekų. Iš granulių paviršiaus vaizdų galime matyti, kad granuliu paviršius turi porų ir tuštumų, kai kurios iš šiu poru yra uždaros, kitos yra susisiekiančios su granulès viduje esančiomis poromis, visų porų sienelès yra glotnios, sienelių medžiaga sustiklëjusi. Mažesnèse granulèse poros ir tuštumos yra išsidėsčiusios chaotiškai, didesnèse granulèse vidinès erdvès struktūra tvarkinga, didžiają dalị užima taisyklingai išsidėsčiusios didelès poros, o erdvè tarp ju yra pripildyta mažesnių poru. Granules sudaro amorfinè fazè, pagal mineraloginę sudèti galima išskirti vieną mineralą kristobalitą. Darbe buvo tiriamos dviejų frakcijų garnulès: $0 / 2$ ir $2 / 4$, taip pat nustatyta $0 / 4$ frakcijos granuliometrinè sudètis. Ištirtos ir nustatytos pagrindinès fizikinès granulių savybės ir savybės, susijusios su vandens poveikiu medžiagai, pateikti mikrostruktūriniai granulių paviršiaus ir vidinès struktūros vaizdai. Pagal ištirtas savybes matyti, kad granulès gali būti pritaikomos vandens valymo technologijose.

Reikšminiai žodžiai: absorbcija, efektyvusis granulių poringumas, putstiklio granulès, granuliu paviršiaus struktūra, poringosios erdvès rezervas. 